\title{
1018 CARRYING CAPACITY ON A MOTOR BICYCLE
}

A G Hemasiri,* J Edirisinghe* Correspondence to Department of Civil Engineering, University of Peradeniya, Peradeniya 20400, Sri Lanka

\subsection{6/ip.2010.029215.1018}

Driver behaviour and attitude contributes significantly towards road traffic accidents and congestion. Due to poor transport facilities, and due to cost, many tend to use motor bicycles for their day to day needs. Sometimes, riders tend to carryout dangerous activities on roads. Here in this picture a rider with a pillion rider carrying a large board risking their lives as well as lives of others on the road. 